Теорія Ймовір. та Матем. Статист. Вип. 76, 2007
Theor. Probability and Math. Statist.

No. 76, 2008, Pages 59-76

S 0094-9000(08)00732-1

Article electronically published on July 14, 2008

\title{
MAXIMAL UPPER BOUNDS FOR THE MOMENTS OF STOCHASTIC INTEGRALS AND SOLUTIONS OF STOCHASTIC DIFFERENTIAL EQUATIONS WITH RESPECT TO FRACTIONAL BROWNIAN MOTION WITH HURST INDEX $H<1 / 2$. II
}

UDC 519.21

\author{
YU. V. KOZACHENKO AND YU. S. MISHURA
}

\begin{abstract}
We study stochastic differential equations with Wiener integral considered with respect to fractional Brownian motion with Hurst index $H<1 / 2$. We prove the existence and uniqueness of a strong solution of the equations and find maximal upper bounds for moments of a solution and its increments. We obtain estimates for the distribution of the supremum of a solution on an arbitrary interval. The modulus of continuity of solutions is found and estimates for the distributions of the norms of solutions are obtained in some Lipschitz spaces.
\end{abstract}

\section{INTRODUCTION}

In this paper, we study properties of solutions of a stochastic differential equation including an additive term represented in the form of a Wiener integral considered with respect to fractional Brownian motion with Hurst parameter $H<1 / 2$. Properties of the integrals of this kind are studied in the first part of this paper (see 2 2). Similar stochastic differential equations with an additive fractional Brownian motion are studied in the paper [1] where the existence and uniqueness of a strong solution is proved.

We show below that solutions of these differential equations belong to the Orlicz space $L_{U}(\Omega)$ of random variables generated by the $N$-function $U(x)=\exp \left\{x^{2}\right\}-1$. This allows us to apply the theory of Orlicz spaces [6] when analyzing the behavior of solutions of stochastic differential equations. We obtain some estimates for the norms of the solutions in the space $L_{U}(\Omega)$.

Further we prove that the supremum of a solution belongs to the same Orlicz space as the solution itself and find estimates for the norm of the supremum. Using the latter result we obtain an estimate for the distribution of the supremum of a solution. It is worthwhile mentioning that the latter estimates have the same rate of growth as in the case of Gaussian processes.

Another topic we study in the paper is the modulus of continuity of solutions. We show that a solution of the equations under consideration belongs with probability one to a certain Lipschitz space and find estimates for the distribution of the norm of the solution in this space.

The paper is organized as follows.

2000 Mathematics Subject Classification. Primary 60G15; Secondary 60H05, 60H10.

Key words and phrases. Fractional Brownian motion, Wiener integral, stochastic differential equation, moment estimates.

Research is partially supported by the NATO grant PST.CLG.9804. 
Conditions for the existence and uniqueness of a solution of a stochastic differential equation are given in Section 2. The proof presented in Section 2 generalizes an analogous proof of the existence and uniqueness of a strong solution of a stochastic differential equation with additive fractional Brownian motion (see [1]). Note that the proof of the existence of a strong solution is nontrivial for $H<1 / 2$.

Upper bounds for moments of a solution and its increments are given in Section 3 .

Estimates for the Orlicz norm of a solution and its increments are studied in Section 4. The estimates are based on the general results obtained in [6].

Bounds for the Orlicz norm of the supremum of a solution are found in Section 5.

Section 6 is devoted to some estimates of the modulus of continuity of a solution. We prove that a solution belongs with probability one to a certain Lipschitz space.

\section{Existence And uniqueness of a STRONG SOlution of A STOChastic}

DIFFERENTIAL EQUATION WITH A WIENER INTEGRAL CONSIDERED WITH RESPECT TO FRACTIONAL BROWNIAN MOTION

Let $t \in \mathbb{R},\left(\Omega, \mathcal{F}, \mathcal{F}_{t}, t \in \mathbb{R}, \mathrm{P}\right)$ be a probability space with filtration,

$$
\left\{B_{t}^{H}, \mathcal{F}_{t}, t \in \mathbb{R}\right\}
$$

a fractional Brownian motion with Hurst index $H \in(0,1 / 2),[0, T] \subset \mathbb{R}$, and let a function $f:[0, T] \rightarrow \mathbb{R}$ be such that $f \in L_{H}^{2}(0, t)$ for all $t \in[0, T]$, while a function $b(t, x):[0, T] \times \mathbb{R} \rightarrow \mathbb{R}$ be measurable with respect to all its arguments. Consider the following stochastic differential equation:

$$
X_{t}=x+\int_{0}^{t} f(s) d B_{s}^{H}+\int_{0}^{t} b\left(s, X_{s}\right) d s, \quad t \in[0, T], x \in \mathbb{R},
$$

where the Wiener integral $I_{t}:=\int_{0}^{t} f(s) d B_{s}^{H}$ is considered with respect to the fractional Brownian motion $B^{H}$ and is defined by

$$
\int_{0}^{t} f(s) d B_{s}^{H}:=\int_{\mathbb{R}}\left(M_{-}^{H} f 1_{(0, t)}\right)(s) d W_{s} .
$$

Here $\left\{W_{t}, \mathcal{F}_{t}, t \in \mathbb{R}\right\}$ is a Wiener process for which $B^{H}$ admits the Mandelbrot-van Ness representation. In what follows we consider a separable modification of the Gaussian process $I_{t}$.

A partial case of equation (11) on the interval $[0, T]$ for the function $f \equiv 1$ is considered in the paper [1]. The existence and uniqueness theorem is proved for a strong solution in [1. We extend this result to equation (1) for the case of more general functions $f$. Our proof follows the lines of the proof in [1], so we only briefly describe the steps and indicate the places where the original proof of 1 requires changes.

Let $p>1 / H, f \in L_{p}(0, T) \cap D_{H, p}(0, T)$ (the corresponding notation is introduced in [2],

$$
G_{p}^{1}(0, t, f):=C_{H}^{(6)}\|f\|_{L_{p}(0, t)} t^{H-1 / p}+C_{H}^{(7)}\|f\|_{D_{H, p}(0, t)} t^{1 / 2-1 / p}
$$

where the constants $C_{H}^{(6)}$ and $C_{H}^{(7)}$ depend on $H$ and $p$ only (their explicit values are given in [2]) and

$$
Y_{t}^{*}:=\sup _{0 \leq s \leq t}\left|Y_{s}\right|
$$

According to Remark 2.5 of [2],

$$
\left\|I_{t}\right\|_{2} \leq G_{p}^{1}(0, t, f)
$$

By Theorem 4.1 of [2],

$$
\left\|I_{T}^{*}\right\|_{2} \leq C_{p, r} G_{p}^{1}(0, T, f)
$$


where $I_{T}^{*}=\sup _{0 \leq t \leq T}\left|I_{t}\right|$,

$$
\begin{gathered}
C_{p, r}= \begin{cases}2^{3 / 2+1 / 2 p}\left(C_{2 p}\right)^{1 / 2 p} & \text { if } r<2 p, \\
\frac{2^{2+1 / r}}{1-p / r}\left(C_{r}\right)^{1 / r} & \text { if } r \geq 2 p,\end{cases} \\
C_{r}=\frac{2^{r / 2}}{\pi^{1 / 2}} \Gamma\left(\frac{r+1}{2}\right),
\end{gathered}
$$

and $\|\cdot\|_{r}=\|\cdot\|_{L_{r}(P)}, r \geq 1$.

Theorem 2.1. Assume that

1) $|f(t)|>0$ for all $t \in[0, T]$,

2) $|b(t, x)| \leq C(1 \wedge|f(t)|)(1+|x|)$,

3) the integral $\int_{0}^{T} \psi(t) d t$ converges for some $r>1$, where $\psi(t)=\|f\|_{L_{1 / H}(0, t)}^{-r}$.

Then equation (11) possesses a unique strong solution on the interval $[0, T]$.

Proof. We split the proof into several steps.

a) Existence of a weak solution. A pair of adapted and almost surely continuous processes $\left(\widetilde{B}^{H}, X\right)$ on the probability space with a filtration $\left(\Omega, \mathcal{F}, \mathcal{F}_{t}, t \in \mathbb{R}, \mathrm{P}\right)$ is called a weak solution of equation (1) if $\widetilde{B}^{H}$ is an $\mathcal{F}_{t}$-fractional Brownian motion, while the process $X$ satisfies equation (1) where $\widetilde{B}^{H}$ substitutes $B^{H}$.

Put

$$
\widetilde{B}_{t}^{H}:=B_{t}^{H}-\int_{0}^{t} f^{-1}(s) b\left(s, x+I_{s}\right) d s
$$

and consider the kernel

$$
K_{H}(t, s)=\Gamma\left(H+\frac{1}{2}\right)^{-1}(t-s)^{H-1 / 2} F\left(H-\frac{1}{2}, \frac{1}{2}-H, H+\frac{1}{2}, 1-\frac{t}{s}\right),
$$

where $F(\cdot, \cdot, \cdot, \cdot)$ is the Gauss hypergeometric function. According to Theorem 2 of [1, the process $\widetilde{B}^{H}$ is a fractional Brownian motion with respect to a filtration $\mathcal{F}_{t}^{B^{H}}$ and a measure $\widetilde{P}$ such that $d \widetilde{P} / d P=\xi_{T}$ if assumptions 1) and 2) hold. Moreover

$$
\xi_{T}=\exp \left\{-\int_{0}^{T} g(s) d W_{s}-\frac{1}{2} \int_{0}^{t} g^{2}(s) d s\right\},
$$

where $W_{t}$ is a Wiener process such that

$$
B_{t}^{H}=\int_{0}^{t} K_{H}(t, s) d W_{s}, \quad g(s)=\left(K_{H}^{-1} \int_{0}^{\cdot} u_{r} d r\right)(s),
$$

and the operator $K_{H}$ is defined by the fractional integrals

$$
\left(K_{H} h\right)(s)=I_{0+}^{2 H} s^{1 / 2-H} I_{0+}^{1 / 2-H} s^{H-1 / 2} h
$$

(see [3]), and $u_{r}=-f^{-1}(r) b\left(r, I_{r}+x\right)$. Similarly to Theorem 3 of [1], the processes $\left(\int_{0}^{t} f(s) d B_{s}^{H}, \widetilde{B}_{t}^{H}\right)$ form a weak solution of equation (1). This can also be proved straightforwardly:

$$
I_{t}=x+\int_{0}^{t} f(s) d \widetilde{B}_{s}^{H}+\int_{0}^{t} b\left(s, x+I_{s}\right) d s .
$$

The only result to be checked is that $\mathrm{E} \xi_{T}=1$. To this end one can check the Novikov condition written in the form $\sup _{0 \leq s \leq T} \operatorname{E} \exp \left(\lambda g_{s}^{2}\right)<\infty$ for some $\lambda>0$. Similarly to formula (15) of [1],

$$
|g(s)|=C_{H} s^{H-1 / 2}\left|\int_{0}^{s}(s-r)^{-1 / 2-H} r^{1 / 2-H} u_{r} d r\right| \leq C_{H} C\left(1+|x|+I_{T}^{*}\right),
$$


and thus one can apply the Fernique theorem [4] on the exponential integrability of a squared seminorm of the Gaussian process $I_{t}, 0 \leq t \leq T$.

b) Uniqueness in distribution and pathwise uniqueness of a weak solution. Let $X_{t}$, $t \in[0, T]$, be a weak solution. Put

$$
\begin{gathered}
v_{s}:=\left(K_{H}^{-1} \int_{0}^{\cdot} f^{-1}(r) b\left(r, X_{r}\right) d r\right)(s) \\
Z_{T}:=\frac{d \widetilde{P}}{d P}=\exp \left\{-\int_{0}^{T} v_{s} d W_{s}-\frac{1}{2} \int_{0}^{T} v_{s}^{2} d s\right\} .
\end{gathered}
$$

Again we need to prove that (3) defines the measure $\widetilde{P}$. It is clear that the required result follows from

$$
\sup _{0 \leq s \leq T} \operatorname{Exp}\left(\lambda v_{s}^{2}\right)<\infty .
$$

Similarly to (2),

$$
\left|v_{s}\right| \leq C_{H} C\left(1+X_{T}^{*}\right) .
$$

According to Lemma 4.1 of [2] the process $I_{t}$ is almost surely continuous in the interval $[0, T]$. For an arbitrary $N>0$, consider $\tau_{N}=\inf \left\{t \geq 0:\left|X_{t}\right| \geq N\right\} \wedge T$. Then

$$
\left|\int_{0}^{t \wedge \tau_{N}} b\left(s, X_{s}\right) d s\right|=\left|\int_{0}^{t} b\left(s, X_{s \wedge \tau_{N}}\right) I\left\{s<\tau_{N}\right\} d s\right| \leq C(1+N) t .
$$

Thus the latter integral is a continuous in $t \in[0, T]$ function. Since

$$
\sup _{0 \leq t \leq T}\left|X_{t \wedge \tau_{N}}\right| \leq|x|+I_{T}^{*}+C \int_{0}^{t \wedge \tau_{N}}\left(1+X_{s}^{*}\right) d s,
$$

we have

$$
\sup _{0 \leq t \leq T}\left|X_{t \wedge \tau_{N}}\right| \leq\left(|x|+I_{T}^{*}+C T\right) e^{C T}
$$

by the Gronwall lemma. Therefore

$$
\sup _{0 \leq t \leq T}\left|X_{t}\right| \leq\left(|x|+\sup _{0 \leq t \leq T}\left|I_{t}\right|+C T\right) e^{C T} .
$$

Moreover the process $X$ is continuous in $t$. We again use the Fernique theorem and obtain a new measure $\widetilde{P}$. The process $\widetilde{W}_{t}:=W_{t}+\int_{0}^{t} v_{s} d s$ is an $\mathcal{F}_{t}$-Wiener process with respect to the measure $\widetilde{P}$. Thus

$$
\begin{aligned}
X_{t} & =x+\int_{0}^{t} f(s) d\left(B_{s}^{H}+\int_{0}^{s} f^{-1}(u) b\left(u, X_{u}\right) d u\right) \\
& =x+\int_{0}^{t} f(s) d\left(\int_{0}^{s} K_{H}(s, u) d \widetilde{W}_{u}\right)=x+\int_{0}^{t} f(s) d \widetilde{B}_{s}^{H},
\end{aligned}
$$

where $\widetilde{B}^{H}$ is a fractional Brownian motion with respect to the measure $\widetilde{P}$. Similarly to Section 3.3 of [1] we check that $X-x$ and $\widetilde{I}_{t}:=\int_{0}^{t} f(s) d \widetilde{B}_{s}^{H}$ have the same distribution with respect to the measure $P$, that is, two arbitrary weak solutions of equation (11) have the same distribution. Since the processes $X_{t}^{1} \wedge X_{t}^{2}$ and $X_{t}^{1} \vee X_{t}^{2}$ also are solutions for given weak solutions $X_{t}^{1}$ and $X_{t}^{2}$, we prove that two arbitrary weak solutions on the same probability space with a filtration coincide almost surely.

c) Krylov's inequality. Let a function $h(s, x):=f^{-1}(s) b(s, x)$ be bounded and let $X$ be a weak solution of equation (11). If assumption 3) of Theorem 2.1 holds, then there 
exists a constant $C>0$ depending on

$$
h:=\sup _{0 \leq t \leq T, x \in \mathbb{R}}|h(t, x)|
$$

and such that

$$
\mathrm{E} \int_{0}^{T} g\left(t, X_{t}\right) d t \leq C\left(\int_{0}^{T} \int_{\mathbb{R}} g^{2}(t, x) d x d t\right)^{1 / 2}
$$

for any nonnegative measurable function $g(t, x):[0, T] \times \mathbb{R} \rightarrow \mathbb{R}$. Indeed, let a measure $\widetilde{P}$ be given by relation (3). Then $X_{t}-x$ has the Gaussian distribution with mean 0 and variance $\sigma_{t}^{2}:=\left\|I_{t}\right\|_{2}^{2}$ with respect to the measure $\widetilde{P}$. Moreover,

$$
\int_{0}^{T} g\left(t, X_{t}\right) d t \leq\left(\widetilde{E} Z_{T}^{-\alpha}\right)^{1 / \alpha}\left(\widetilde{E} \int_{0}^{T} g\left(t, X_{t}\right)^{\beta} d t\right)^{1 / \beta}
$$

by the Hölder inequality with $1 / \alpha+1 / \beta=1$. Note that

$$
\begin{aligned}
\widetilde{E} Z_{T}^{-\alpha} & =\widetilde{E} \exp \left(\alpha \int_{0}^{T} v_{s} d W_{s}+\frac{\alpha}{2} \int_{0}^{T} v_{s}^{2} d s\right) \\
& =\widetilde{E} \exp \left(\alpha \int_{0}^{T} v_{s} d \widetilde{W}_{s}-\frac{\alpha^{2}}{2} \int_{0}^{T} v_{s}^{2} d s\right) \exp \left\{\frac{\alpha^{2}+\alpha}{2} \int_{0}^{T} v_{s}^{2} d s\right\}<\infty,
\end{aligned}
$$

since the expectation of the first factor equals 1 in view of bounds (4)-(5), while the second factor is finite in view of the same bounds and by taking into account that the functions $h$ and $v_{s}$ are bounded.

Now let $1 / \gamma+1 / \gamma^{\prime}=1$ and $\gamma \beta=2$. Then

$$
\begin{aligned}
& \widetilde{E} \int_{0}^{T} g\left(t, X_{t}\right)^{\beta} d t=\int_{0}^{T} \frac{1}{\sqrt{2 \pi} \sigma_{t}} \int_{\mathbb{R}} g(t, y)^{\beta} \exp \left\{-\frac{(y-x)^{2}}{2 \sigma_{t}^{2}}\right\} d y d t \\
& \leq \frac{1}{\sqrt{2 \pi}}\left(\int_{0}^{T} \int_{\mathbb{R}} g(t, y)^{\gamma \beta} d y d t\right)^{1 / \gamma}\left(\int_{0}^{T} \int_{\mathbb{R}} \exp \left\{-\frac{\gamma^{\prime}(y-x)^{2}}{2 \sigma_{t}^{2}}\right\} \sigma_{t}^{-\gamma^{\prime}} d y d t\right)^{1 / \gamma^{\prime}} \\
& \quad=\frac{1}{\sqrt{2 \pi}}\left(\int_{0}^{T} \int_{\mathbb{R}} g(t, y)^{2} d y d t\right)^{1 / \gamma}\left(\int_{0}^{T} \int_{\mathbb{R}} e^{-\gamma^{\prime} z^{2}} \sigma_{t}^{1-\gamma^{\prime}} d z d t\right)^{1 / \gamma^{\prime}} \\
& \leq C\left(\int_{0}^{T} \int_{\mathbb{R}} g(t, y)^{2} d y d t\right)^{1 / \gamma}\left(\int_{0}^{T} \sigma_{t}^{-\beta /(2-\beta)} d t\right)^{(2-\beta) / 2} .
\end{aligned}
$$

Finally we set $\beta /(2-\beta)=r>1$, that is, we choose

$$
\beta=\frac{2 r}{1+r}, \quad \frac{1}{\alpha}=1-\frac{1}{\beta}, \quad \gamma=1+\frac{1}{r} .
$$

Then $\gamma^{-1}=r /(1-r)$. Using the inequality $\left\|I_{t}\right\|_{2} \geq C(H)\|f\|_{L_{1 / H}(0, t)}$ proved in [5] and assumption 3) we obtain

$$
\int_{0}^{T} \sigma_{t}^{-r} d t<\infty .
$$

Then inequality (6) follows from (7)-(9).

d) Existence of a strong solution. Taking into account estimate ([6), the proof of this part is similar to that in [1].

Namely we assume that the coefficient $b$ is such that

$$
|b(s, x)| \leq C_{1}(|f(s)| \wedge 1)
$$


YU. V. KOZACHENKO AND YU. S. MISHURA

for some constant $C_{1}>0$. This means that the functions $|b(s, x)|$ and $\left|f^{-1}(s) b(s, x)\right|$ are bounded. Similarly to Proposition 7 of [1] we prove that if a sequence of functions $b_{n}(t, x)$ satisfies inequality (10) with the same constant $C_{1}$ for all functions, if $b_{n}(t, x) \rightarrow b(t, x)$ for almost all $(t, x) \in[0, T] \times \mathbb{R}$, and if equation (11) with $b_{n}(t, x)$ possesses a solution $X_{n}$ such that $X_{n}(t) \rightarrow X(t)$ almost surely for all $t \in[0, T]$, then $X(t)$ is a solution of equation (11) with $b(t, x)$. The proof of this result uses inequality (6) .

Further we use the comparison principle and follow the lines of the proof of Theorem 8 of [1. As a result we prove that if $|b(s, x)| \leq C_{1}(|f(s)| \wedge 1)(1+|x|)$, then equation (1) has a strong solution (then Step b) proves the uniqueness).

\section{Bounds FOR MOMEnTs of A SOLUtion of EQUATION (1)}

As before let $\tau_{N}=\inf \left\{t>0:\left|X_{t}\right|>N\right\} \wedge T$ and let $X_{t}$ be a solution of equation (1). We checked already that $X_{t}$ is continuous in $t$, whence we derive $\left|X_{t \wedge \tau_{N}}\right| \leq N$.

Now

$$
\left|X_{t \wedge \tau_{N}}\right| \leq|x|+\left|I_{t \wedge \tau_{N}}\right|+C \int_{0}^{t}\left(1+\left|X_{s \wedge \tau_{N}}\right|\right) d s
$$

and

$$
\begin{aligned}
\mathrm{E}\left|X_{t \wedge \tau_{N}}\right|^{r} & \leq 3^{r}\left(|x|^{r}+C^{r} \mathrm{E}\left(\int_{0}^{t}\left(1+\left|X_{s \wedge \tau_{N}}\right|\right) d s\right)^{r}+\mathrm{E}\left|I_{t \wedge \tau_{N}}\right|^{r}\right) \\
& \leq 3^{r}|x|^{r}+(3 C)^{r} \mathrm{E} \int_{0}^{t}\left(1+\left|X_{s \wedge \tau_{N}}\right|\right)^{r} d s \cdot t^{r-1}+3^{r} \mathrm{E}\left|I_{t \wedge \tau_{N}}\right|^{r} \\
& \leq 3^{r}|x|^{r}+(6 C)^{r} t^{r}+(6 C)^{r} \mathrm{E} \int_{0}^{t}\left|X_{s \wedge \tau_{N}}\right|^{r} d s \cdot t^{r-1}+3^{r} \mathrm{E}\left|I_{t \wedge \tau_{N}}\right|^{r} \\
& \leq g(t)+(6 C)^{r} t^{r-1} \int_{0}^{t}\left|X_{s \wedge \tau_{N}}\right|^{r} d s
\end{aligned}
$$

for all $r>1$, where

$$
g(t)=3^{r}|x|^{r}+(6 C)^{r} t^{r}+3^{r} \mathrm{E}\left|I_{t}^{*}\right|^{r} .
$$

Applying the Gronwall inequality we get

$$
\mathrm{E}\left|X_{t \wedge \tau_{N}}\right|^{r} \leq g(t)\left[1+C_{1} t^{r} \exp \left\{\frac{C_{i} t^{r}}{r}\right\}\right]
$$

where $C_{1}=(6 C)^{r}$. Passing to the limit as $N \rightarrow \infty$ we obtain

$$
\mathrm{E}\left|X_{t}\right|^{r} \leq g(t)\left[1+C_{1} t^{r} \exp \left\{\frac{C_{1} t^{r}}{r}\right\}\right]
$$

By Theorem 4.1 of [2] we have

$$
\left\|I_{t}^{*}\right\|_{r} \leq C_{p, r} G_{p}^{1}(0, t, f) .
$$

Thus (11)-(14) imply that

$$
\mathrm{E}\left|X_{t}\right|^{r} \leq g(t)\left(1+C_{1} t^{r} \exp \left\{\frac{C_{1} t^{r}}{r}\right\}\right)
$$

where $g(t)=3^{r}|x|^{r}+(6 C)^{r} t^{r}+3^{r} C_{p, r}^{r}\left(G_{p}^{1}(0, t, f)\right)^{r}$.

Estimate (15) implies that $\mathrm{E}\left|X_{t}\right|^{r}$ is finite. Estimate (15) can also be used to decrease the constant in the expression for $g(t)$.

Indeed, if $\mathrm{E}\left|X_{t}\right|^{r}<\infty$, then one can write

$$
\mathrm{E}\left|X_{t}\right|^{r} \leq \mathrm{E}\left(|x|+\left|I_{t}\right|+C\left(\int_{0}^{t}\left(1+\left|X_{s}\right|\right) d s\right)\right)^{r} \leq g_{1}(t)+C_{1} t^{r-1} \int_{0}^{t} \mathrm{E}\left|X_{s}\right|^{r} d s
$$


instead of bound (11), where

$$
g_{1}(t)=(3|x|)^{r}+C_{1} t^{r}+3^{r} \sup _{0 \leq s \leq t} \mathrm{E}\left|I_{s}\right|^{r} \leq(3|x|)^{r}+C_{1} t^{r}+\widetilde{C_{r}}\left(G^{1}(0, t, f)\right)^{r}
$$

by estimate (9) of [2],

$$
G^{1}(0, t, f):=C_{H}^{(6)}\|f\|_{L_{p}(0, t)} \cdot t^{H-1 / p}+C_{H}^{(7)}\|f\|_{D_{H}(0, t)},
$$

and $\widetilde{C_{r}}=3^{r} C_{r}$. Therefore

$$
\mathrm{E}\left|X_{t}\right|^{r} \leq g_{1}(t)\left(1+C_{1} t^{r} \exp \left\{\frac{C_{1} t^{r}}{r}\right\}\right)
$$

by the Gronwall inequality.

For $0 \leq t<t^{\prime} \leq T$, we proceed in an analogous way:

$$
\begin{aligned}
\mathrm{E} \mid X_{t} & -\left.X_{t^{\prime}}\right|^{r} \leq(2 C)^{r} \mathrm{E}\left(\int_{t}^{t^{\prime}}\left(1+\left|X_{s}\right|\right) d s\right)^{r}+2^{r} \mathrm{E}\left|\int_{t}^{t^{\prime}} f(s) d B_{s}^{H}\right|^{r} \\
\leq & (4 C)^{r}\left(\left(t^{\prime}-t\right)^{r}+\left(t^{\prime}-t\right)^{r-1} \int_{t}^{t^{\prime}} \mathrm{E}\left|X_{s}\right|^{r} d s\right)+2^{r} \mathrm{E}\left|\int_{t}^{t^{\prime}} f(s) d B_{s}^{H}\right|^{r} \\
\leq & (4 C)^{r}\left(1+g_{1}(T)\left(1+C_{1} T^{r} \exp \left\{\frac{C_{1} T^{r}}{r}\right\}\right)\right)\left(t^{\prime}-t\right)^{r} \\
& +2^{r} C_{r}\left(G^{2}\left(t, t^{\prime}, f\right)\right)^{r},
\end{aligned}
$$

where $G^{2}\left(t, t^{\prime}, f\right)=C_{H}^{(6)}\|f\|_{L_{p}\left(t, t^{\prime}\right)} \cdot\left(t^{\prime}-t\right)^{H-1 / p}+C_{H}^{(7)}\|f\|_{D_{H}\left(t, t^{\prime}\right)}$.

In particular, let $f \in C^{\beta}[0, T]$ for $H-1 / 2+\beta>0$ and $0<\beta<1$. Then

$$
\begin{gathered}
\|f\|_{L_{p}\left(t, t^{\prime}\right)} \cdot\left(t^{\prime}-t\right)^{H-1 / p} \leq\|f\|_{C^{\beta}[0, T]}\left(t^{\prime}-t\right)^{H} \\
\|f\|_{D_{H}\left(t, t^{\prime}\right)} \leq\|f\|_{C^{\beta}[0, T]}\left(\int_{t}^{t^{\prime}}\left(\int_{x}^{t^{\prime}}(t-x)^{H+\beta-3 / 2} d t\right)^{2} d x\right)^{1 / 2} \\
\leq C_{H, \beta}^{1}\|f\|_{C^{\beta}[0, T]}\left(t^{\prime}-t\right)^{H+\beta}
\end{gathered}
$$

where $C_{H, \beta}^{1}=(H+\beta-1 / 2)^{-1}(2 H+2 \beta)^{-1 / 2}$, whence

$$
\begin{aligned}
\mathrm{E}\left|X_{t}-X_{t^{\prime}}\right|^{r} \leq & (4 C)^{r}\left(1+g_{1}(T)\left(1+C_{1} T^{r} \exp \left\{\frac{C_{1} T^{r}}{r}\right\}\right)\right)\left(t^{\prime}-t\right)^{r} \\
& +2^{r} C_{r}\left(C_{H, \beta, T}\right)^{r}\left(t^{\prime}-t\right)^{r H}
\end{aligned}
$$

for $C_{H, \beta, T}=\left(C_{H}^{(6)}+C_{H}^{(7)} C_{H, \beta}^{1} T^{\beta}\right) \cdot\|f\|_{C^{\beta}(0, T)}$.

Estimates (16) and (17) can be improved further by dividing the interval $[0, T]$ appropriately. Namely we choose $t_{0}:=(6 C)^{-1}$. Then

$$
\mathrm{E}\left|X_{t}\right|^{r} \leq g_{1}(t)+6 C \int_{0}^{t} \mathrm{E}\left|X_{s}\right|^{r} d s
$$

for $0 \leq t \leq t_{0}$ according to (16). Hence

$$
\mathrm{E}\left|X_{t}\right|^{r} \leq g_{1} \cdot e^{6 C t} \leq e \cdot g_{1}, \quad 0 \leq t \leq t_{0},
$$

by the Gronwall inequality, where $g_{1}=3^{r}|x|^{r}+1+\widetilde{C_{r}}\left(G^{1}(0, T, f)\right)^{r}$. 
In particular, $\mathrm{E}\left|X_{t_{0}}\right|^{r} \leq e g_{1}$. Further,

$$
\begin{aligned}
\mathrm{E}\left|X_{t}\right|^{r} \leq & 3^{r} \mathrm{E}\left|X_{t_{0}}\right|^{r}+3^{r} \mathrm{E}\left|\int_{t_{0}}^{t} f(s) d B_{s}^{H}\right|^{2}+(6 C)^{r}\left(t-t_{0}\right)^{r} \\
& +(6 C)^{r}\left(t-t_{0}\right)^{r-1} \mathrm{E} \int_{t_{0}}^{t}\left|X_{s}\right|^{r} d s \\
\leq & 3^{r} g_{1} e+\widetilde{C_{r}}\left(G^{1}(0, T, f)\right)^{r}+1+6 C \int_{t_{0}}^{t}\left|X_{s}\right|^{r} d s
\end{aligned}
$$

for $t_{0} \leq t_{1} \leq 2 t_{0}$, whence

$$
\mathrm{E}\left|X_{t}\right|^{r} \leq g_{2} e^{6 C\left(t-t_{0}\right)} \leq g_{2} e
$$

for the constant $g_{2}=3^{r} g_{1} e+\widetilde{C_{r}}\left(G^{1}(0, T, f)\right)^{r}+1$.

Now we use induction for $k t_{0} \leq t \leq(k+1) t_{0}$ and get

$$
\mathrm{E}\left|X_{t}\right|^{r} \leq g_{k+1} e
$$

where $g_{k+1} \leq 3^{r} g_{k} e+B_{r} \leq \cdots \leq\left(3^{r} e\right)^{k}\left(g_{1}+B_{r}\right)$ and $B_{r}=\widetilde{C_{r}}\left(G^{1}(0, T, f)\right)^{r}+1$.

The total number of the steps described above does not exceed $k=\left[T / t_{0}\right]+1 \leq 6 C T+1$ on the interval $[0, T]$.

Thus

$$
\begin{aligned}
\mathrm{E}\left|X_{t}\right|^{r} & \leq\left(3^{r} e\right)^{6 C T+1}\left(g_{1}+B_{r}\right) \\
& \leq(3 e)^{(6 C T+1) r}\left(3^{r}|x|^{r}+2+2 \widetilde{C_{r}}\left(G^{1}(0, T, f)\right)^{r}\right)
\end{aligned}
$$

for all $0 \leq t \leq T$. Similarly to (18) we prove that

$$
\mathrm{E}\left|X_{t}-X_{t^{\prime}}\right|^{r} \leq(4 C)^{r} D_{r}\left(t^{\prime}-t\right)^{r}+2^{r} C_{r}\left(G^{2}\left(t, t^{\prime}, f\right)\right)^{r},
$$

where

$$
\begin{aligned}
D_{r} & =1+(3 e)^{(6 C T+1) r}\left(g_{1}+B_{r}\right) \\
& =1+(3 e)^{(6 C T+1) r}\left(2+(3|x|)^{r}+2 \widetilde{C_{r}}\left(G^{1}(0, T, f)\right)^{r}\right) .
\end{aligned}
$$

If $f \in C^{\beta}(0, T)$ for $0<\beta<1$ and $H+\beta>1 / 2$, then

$\mathrm{E}\left|X_{t}-X_{t^{\prime}}\right|^{r} \leq(4 C)^{r}\left(1+(3 e)^{(6 C T+1) r}\left(g_{1}+B_{r}\right)\right)\left(t^{\prime}-t\right)^{r}+2^{r} C_{r}\left(C_{H, \beta, T}\right)^{r}\left(t^{\prime}-t\right)^{H r}$, that is,

$$
\mathrm{E}\left|X_{t}-X_{t^{\prime}}\right|^{r} \leq(4 C)^{r} D_{r}\left(t^{\prime}-t\right)^{r}+2^{r} C_{r}\left(C_{H, \beta, T}\right)^{r}\left(t^{\prime}-t\right)^{H r} .
$$

4. Bounds For the nORMS OF a SOlution in Orlicz SPACES

Let $U(x)=\exp \left\{x^{2}\right\}-1$ and let $\{\Omega, \mathcal{F}, \mathrm{P}\}$ be a probability space.

Definition 4.1. The family of random variables $\xi$ for which there exists a constant $C_{\xi}>0$ such that

$$
\mathrm{E} U\left(\frac{\xi}{C_{\xi}}\right)<\infty
$$

is called the Orlicz space $L_{U}(\Omega)$ generated by the function $U(x)$.

Theorem 4.1 ([6]). The Orlicz space $L_{U}(\Omega)$ is a Banach space with respect to the Luxemburg norm

$$
\|\xi\|_{U}=\inf \left\{r>0: \operatorname{E} \exp \left\{\frac{\xi^{2}}{r^{2}}\right\} \leq 2\right\} .
$$

Let $\mathbb{T}$ be a set of parameters. 
Definition 4.2. We say that a stochastic process $Y=\left\{Y_{t}, t \in \mathbb{T}\right\}$ belongs to the space $L_{U}(\Omega)$ if for an arbitrary $t \in \mathbb{T}$ the random variable $Y_{t}$ belongs to $L_{U}(\Omega)$.

In what follows we use the following notation:

$$
a:=(3 e)^{6 C T+1}, \quad A:=3|x| a, \quad B:=3 a G^{1}(0, T, f), \quad B_{1}=B \sqrt{2},
$$

$\varepsilon_{0}:=\max \{B, a \sqrt{e}, A \sqrt{e}\}$, and $R:=(3+2 \sqrt{2}) \exp \left\{\varepsilon_{0}^{2} / B_{1}^{2}\right\}$.

Theorem 4.2. Let the assumptions of Theorem 2.1 hold. Let $X_{t}$ be a solution of equation (11) and $t \in[0, T]$. Then

$$
\mathrm{P}\left\{\left|X_{t}\right|>\varepsilon\right\} \leq R \exp \left\{-\frac{\varepsilon^{2}}{2 B^{2}}\right\}
$$

for all $\varepsilon>0$.

Proof. Inequality (19) implies for $r \geq 1$ that

$$
\mathrm{E}\left|X_{t}\right|^{r} \leq A^{r}+2 a^{r}+\frac{2 B_{1}^{r}}{\sqrt{\pi}} \Gamma\left(\frac{r+1}{2}\right) .
$$

Using Stirling's formula,

$$
\Gamma(u)=\sqrt{2 \pi} u^{u-1 / 2} e^{-u} e^{\theta(u)} \quad \text { for } \theta(u)<\frac{1}{12 u}, u \geq 1,
$$

we obtain

$$
\begin{aligned}
\Gamma\left(\frac{r+1}{2}\right) & \leq \sqrt{2 \pi}\left(\frac{r+1}{2}\right)^{r / 2} \cdot \exp \left\{-\frac{r+1}{2}\right\} \exp \left\{\frac{1}{6(r+1)}\right\} \\
& =\sqrt{2 \pi} r^{r / 2}(2 e)^{-r / 2}(1+1 / r)^{r / 2} \exp \left\{-\frac{1}{2}+\frac{1}{6(r+1)}\right\} .
\end{aligned}
$$

It is easy to check that

$$
h(r):=(1+1 / r)^{r / 2} \exp \left\{-\frac{1}{2}+\frac{1}{6(r+1)}\right\} \leq 1
$$

for $r \geq 1$. Indeed,

$$
\begin{aligned}
\ln h(r) & =\frac{r}{2} \ln \left(1+\frac{1}{r}\right)-\frac{1}{2}+\frac{1}{6(r+1)} \\
& \leq \frac{r}{2}\left(\frac{1}{r}-\frac{1}{2 r^{2}}+\frac{1}{3 r^{3}}\right)-\frac{1}{2}+\frac{1}{6(r+1)}=\frac{2-r-r^{2}}{12(r+1) r^{2}} \leq 0
\end{aligned}
$$

for $r \geq 1$, that is,

$$
\Gamma\left(\frac{r+1}{2}\right) \leq \sqrt{2 \pi}(2 e)^{-r / 2} r^{r / 2} .
$$

Now inequalities (23) and (24) imply that

$$
\mathrm{E}\left|X_{t}\right|^{r} \leq A^{r}+2 a^{r}+2 \sqrt{2} D^{r} r^{r / 2}
$$

where $D=B_{1} / \sqrt{2 e}$.

The latter bound together with the Chebyshev inequality yields

$$
\mathrm{P}\left\{\left|X_{t}\right| \geq \varepsilon\right\} \leq \frac{\mathrm{E}\left|X_{t}\right|^{r}}{\varepsilon^{r}} \leq\left(\frac{A}{\varepsilon}\right)^{r}+2\left(\frac{a}{\varepsilon}\right)^{r}+2 \sqrt{2}\left(\frac{D}{\varepsilon}\right)^{r} \cdot r^{r / 2} .
$$


Set $r=(\varepsilon / D)^{2} / e$ in (26) for $\varepsilon>D \sqrt{e}$. Then

$$
\begin{aligned}
\mathrm{P}\left\{\left|X_{t}\right| \geq \varepsilon\right\} \leq & \left(\frac{A}{\varepsilon}\right)^{(\varepsilon / D)^{2} / e}+2\left(\frac{a}{\varepsilon}\right)^{(\varepsilon / D)^{2} / e}+2 \sqrt{2} \exp \left\{-\left(\frac{\varepsilon}{D}\right)^{2} \frac{1}{2 e}\right\} \\
= & \exp \left\{\left(\ln \frac{A}{\varepsilon}\right)\left(\frac{\varepsilon}{D}\right)^{2} \frac{1}{e}\right\}+2 \exp \left\{\left(\ln \frac{a}{\varepsilon}\right)\left(\frac{\varepsilon}{D}\right)^{2} \frac{1}{e}\right\} \\
& +2 \sqrt{2} \exp \left\{-\left(\frac{\varepsilon}{D}\right)^{2} \frac{1}{2 e}\right\} .
\end{aligned}
$$

Let $\ln (A / \varepsilon) \leq-\frac{1}{2}$ and $\ln (a / \varepsilon) \leq-\frac{1}{2}$. These inequalities hold if $\varepsilon \geq \max (a, A) \sqrt{e}$, that is, if

$$
\varepsilon \geq \sqrt{e} a \cdot \max (1,3|x|)
$$

Since $\varepsilon>D \sqrt{e}$, we have

$$
\mathrm{P}\left\{\left|X_{t}\right| \geq \varepsilon\right\} \leq(3+2 \sqrt{2}) \cdot \exp \left\{-\frac{\varepsilon^{2}}{2 e D^{2}}\right\}=(3+2 \sqrt{2}) \cdot \exp \left\{-\frac{\varepsilon^{2}}{2 B^{2}}\right\} .
$$

This means that inequality (29) holds for $\varepsilon \geq \varepsilon_{0}$.

Since $\exp \left\{\varepsilon_{0}^{2} /\left(2 B^{2}\right)\right\}>1$, bound (29) implies inequality (22) for all $\varepsilon>0$.

Theorem 4.3. Let the assumptions of Theorem 2.1 hold. Assume that $X_{t}$ is a solution of equation (11) and $t \in[0, T]$. Then the random variable $X_{t}$ belongs to the Orlicz space $L_{U}(\Omega)$ and its Orlicz norm in $L_{U}(\Omega)$ is such that

$$
\left\|X_{t}\right\|_{U} \leq \sqrt{2}(R+1) B .
$$

Proof. Theorem 4.3 follows from Theorem 4.2 and from the following auxiliary result, which is a particular case of Theorem 2.3.4 in [6].

Lemma 4.1. Let $\xi$ be a random variable such that

$$
\mathrm{P}\{|\xi|>\varepsilon\} \leq C_{1} \exp \left\{-\frac{\varepsilon^{2}}{2 C_{2}^{2}}\right\}
$$

for all $\varepsilon>0$, where $C_{i}>0, i=1,2$. Then $\xi \in L_{U}(\Omega)$ and moreover $\|\xi\|_{U} \leq \sqrt{2}\left(1+C_{1}\right) C_{2}$.

We introduce the following notation:

$$
\begin{gathered}
E_{1}:=2 \sqrt{2} C_{H, \beta, T}, \\
F_{1}:=\frac{E_{1}}{\sqrt{2 e}}, \quad F_{2}:=4 C \frac{B_{1}}{\sqrt{2 e}} T^{1-H}, \quad F_{3}:=4 C(1+2 a+A) T^{1-H}, \\
F_{4}:=F_{1}+F_{2}, \quad F_{5}:=(2 \sqrt{2}+1) \exp \left\{\frac{\max \left(F_{3}, F_{4}\right)}{2 F_{4}^{2}}\right\}, \quad F_{6}:=F_{4} \sqrt{e} .
\end{gathered}
$$

Theorem 4.4. Let $\left\{X_{t}, t \in[0, T]\right\}$ be a solution of equation (11), where $f \in C^{\beta}[0, T]$ and $H+\beta-1 / 2>0$.

Then

$$
\mathrm{P}\left\{\left|X_{t^{\prime}}-X_{t}\right| \geq \varepsilon\right\} \leq F_{5} \exp \left\{-\frac{\varepsilon^{2}}{2 F_{6}^{2}\left(t^{\prime}-t\right)^{2 H}}\right\}
$$

and

$$
\left\|X_{t^{\prime}}-X_{t}\right\|_{U} \leq \sqrt{2}\left(1+F_{5}\right) F_{6}\left(t^{\prime}-t\right)^{H}
$$

for all $\varepsilon>0$ and $0 \leq t<t^{\prime} \leq T$. 
Proof. Inequality (32) follows from (31) and Theorem 4.3. Thus it remains to prove inequality (31). Inequalities (21) and (24) imply that

$$
\begin{aligned}
\mathrm{E}\left|X_{t^{\prime}}-X_{t}\right|^{r} \leq & \frac{1}{\sqrt{\pi}} E_{1}^{r} \Gamma\left(\frac{r+1}{2}\right)\left(t^{\prime}-t\right)^{r H} \\
& +(4 C)^{r}\left(1+2 a^{r}+A^{r}+\frac{2}{\sqrt{\pi}} B_{1}^{r} \Gamma\left(\frac{r+1}{2}\right)\right)\left(t^{\prime}-t\right)^{r} \\
\leq & \sqrt{2} F_{1}^{r} r^{r / 2}\left(t^{\prime}-t\right)^{r H}+2 \sqrt{2} F_{2}^{r} r^{r / 2}\left(t^{\prime}-t\right)^{r H}+F_{3}^{r}\left(t^{\prime}-t\right)^{r H} .
\end{aligned}
$$

Thus

$$
\begin{aligned}
\mathrm{P}\left\{\left|X_{t^{\prime}}-X_{t}\right| \geq \varepsilon\right\} & \leq\left(\left(\sqrt{2}\left(\frac{F_{1}}{\varepsilon}\right)^{r}+2 \sqrt{2}\left(\frac{F_{2}}{\varepsilon}\right)^{r}\right) r^{r / 2}+\left(\frac{F_{3}}{\varepsilon}\right)^{r}\right)\left(t^{\prime}-t\right)^{r H} \\
& \leq\left(2 \sqrt{2}\left(\frac{F_{4}}{\varepsilon}\right)^{r} r^{r / 2}+\left(\frac{F_{3}}{\varepsilon}\right)^{r}\right)\left(t^{\prime}-t\right)^{r H}
\end{aligned}
$$

for all $\varepsilon>0$. If one substitutes

$$
r=\frac{1}{e}\left(\frac{\varepsilon}{\left(t^{\prime}-t\right)^{H} F_{4}}\right)^{2}
$$

in the latter inequality, then

$$
\begin{aligned}
& \mathrm{P}\left\{\left|X_{t^{\prime}}-X_{t}\right| \geq \varepsilon\right\} \\
& \quad \leq 2 \sqrt{2} \exp \left\{-\frac{\varepsilon^{2}}{2\left(t^{\prime}-t\right)^{2 H} F_{6}^{2}}\right\}+\exp \left\{\ln \left(\frac{F_{3}}{\varepsilon}\left(t^{\prime}-t\right)^{H}\right) \cdot \frac{\varepsilon^{2}}{2\left(t^{\prime}-t\right)^{2 H} F_{6}^{2}}\right\}
\end{aligned}
$$

for $r \geq 1$, that is, for $\varepsilon \geq\left(t^{\prime}-t\right)^{H} F_{6}$.

If

$$
\ln \left(\frac{F_{3}}{\varepsilon}\left(t^{\prime}-t\right)^{H}\right) \leq-\frac{1}{2}
$$

that is, if $\varepsilon \geq \sqrt{e}\left(t^{\prime}-t\right)^{H} F_{3}$, then

$$
\begin{aligned}
\mathrm{P}\left\{\left|X_{t^{\prime}}-X_{t}\right| \geq \varepsilon\right\} & \leq(2 \sqrt{2}+1) \exp \left\{-\frac{\varepsilon^{2}}{2\left(t^{\prime}-t\right)^{2 H} F_{6}^{2}}\right\} \\
& \leq F_{5} \exp \left\{-\frac{\varepsilon^{2}}{2\left|t^{\prime}-t\right|^{2 H} F_{6}^{2}}\right\}
\end{aligned}
$$

for $\varepsilon \geq \varepsilon_{0}$, where $\varepsilon_{0}:=\max \left(F_{3}, F_{4}\right) \sqrt{e}\left(t^{\prime}-t\right)^{H}$. Otherwise, if $0<\varepsilon \leq \varepsilon_{0}$, then

$$
\begin{aligned}
\mathrm{P}\left\{\left|X_{t^{\prime}}-X_{t}\right| \geq \varepsilon\right\} & \leq(2 \sqrt{2}+1) \exp \left\{\frac{\varepsilon_{0}^{2}}{2\left(t^{\prime}-t\right)^{2 H} F_{6}^{2}}\right\} \exp \left\{-\frac{\varepsilon^{2}}{2\left(t^{\prime}-t\right)^{2 H} F_{6}^{2}}\right\} \\
& =F_{5} \exp \left\{-\frac{\varepsilon^{2}}{2\left(t^{\prime}-t\right)^{2 H} F_{6}^{2}}\right\} .
\end{aligned}
$$

Put $F_{7}:=\left(1+F_{5}\right) F_{6}$.

Corollary 4.1. Let $\left\{X_{t}, t \in[0, T]\right\}$ be a solution of equation (11) and let the assumptions of Theorem 4.1 hold. Then

$$
\mathrm{E} \exp \left\{\lambda\left|X_{t}-X_{t^{\prime}}\right|\right\} \leq 2 \exp \left\{\frac{\lambda^{2}}{2} F_{7}^{2}\left(t^{\prime}-t\right)^{2 H}\right\}
$$

for all $\lambda \in \mathbb{R}$ and $0 \leq t<t^{\prime} \leq T$.

Corollary 4.1 follows from bound (32) and the following auxiliary result being a particular case of Lemma 2.3.4 in the book 6 . 
Lemma 4.2. If a random variable $\xi$ belongs to the space $L_{U}(\Omega)$, where

$$
U(x)=\exp \left\{x^{2}\right\}-1,
$$

then

$$
\mathrm{E} \exp \{\lambda|\xi|\} \leq 2 \exp \left\{\frac{\lambda^{2}\|\xi\|_{U}^{2}}{4}\right\}
$$

for all $\lambda \in \mathbb{R}$.

5. The distribution of the supremum of the process $X$ in the interval $[0, T]$

First we recall some notions of the theory of stochastic processes belonging to Orlicz spaces.

Let $\mathbb{T}$ be an infinite set of parameters and let $Y=\left\{Y_{t}, t \in \mathbb{T}\right\}$ be a real stochastic process belonging to the space $L_{U}(\Omega)$, where $U(x)=\exp \left\{x^{2}\right\}-1$. Assume that $\sup _{t \in \mathbb{T}}\left\|Y_{t}\right\|_{U}<\infty$ and let $\rho_{Y}(t, s)=\left\|Y_{t}-Y_{s}\right\|_{U}$ denote a pseudometric on $\mathbb{T}$.

Let the space $\left(\mathbb{T}, \rho_{Y}\right)$ be separable and let the process $Y_{t}$ be separable on $\left(\mathbb{T}, \rho_{Y}\right)$, where $N(\varepsilon)$ is the metric capacity of $\left(\mathbb{T}, \rho_{Y}\right)$, that is, the minimal number of closed balls whose radii do not exceed $\varepsilon$ and that cover $\left(\mathbb{T}, \rho_{Y}\right)$. Note that $N(\varepsilon) \rightarrow \infty$ as $\varepsilon \rightarrow 0$.

The following result is a particular case of Theorem 3.3.4 in [6].

Theorem 5.1. If

$$
\int_{0}^{\varepsilon_{0}}(\ln (1+N(\varepsilon)))^{1 / 2} d \varepsilon<\infty
$$

where $\varepsilon_{0}=\sup _{t, s \in \mathbb{T}} \rho_{Y}(t, s)$, then the random variable $\sup _{t \in \mathbb{T}}\left|Y_{t}\right|$ belongs to the space $L_{U}(\Omega)$ and moreover

$$
\left\|\sup _{t \in \mathbb{T}}\left|Y_{t}\right|\right\|_{U} \leq K:=\inf _{t \in \mathbb{T}}\left\|Y_{t}\right\|_{U}+\frac{e^{2}}{\theta(1-\theta)} \int_{0}^{\theta \varepsilon_{0}}(\ln (1+N(\varepsilon)))^{1 / 2} d \varepsilon<\infty,
$$

where $\theta$ is such that $0<\theta<1$ and $N\left(\theta \varepsilon_{0}\right)>e^{2}-1$.

Remark 5.1. Theorem 5.1 holds if another function $N_{1}(\varepsilon)$ such that $N_{1}(\varepsilon) \geq N(\varepsilon)$ substitutes $N(\varepsilon)$ in condition (34).

Remark 5.2. If assumptions of Theorem 5.1 hold for all $\varepsilon>0$, then

$$
\mathrm{P}\left\{\sup _{t \in \mathbb{T}}\left|Y_{t}\right|>\varepsilon\right\} \leq 2 \exp \left\{-\frac{\varepsilon^{2}}{K^{2}}\right\},
$$

where the constant $K$ is defined by (35).

Inequality (36) follows from the following result: if $\xi \in L_{U}(\Omega)$, then

$$
\mathrm{P}\{|\xi|>\varepsilon\} \leq 2 \exp \left\{-\frac{\varepsilon^{2}}{\|\xi\|_{U}}\right\}
$$

for all $\varepsilon>0$. In its turn, inequality (37) is a particular case of Theorem 3.3.4 in [6].

Theorem 5.2. Let $\left\{Y_{t}, t \in[a, b]\right\},-\infty<a<b<\infty$, be a separable stochastic process belonging to the space $L_{U}(\Omega)$. Assume that there exists a function $\sigma(h), 0 \leq h \leq b-a$, such that $\sigma(h)$ is increasing, continuous, $\sigma(h) \rightarrow 0$ as $h \rightarrow 0$, and

$$
\sup _{|t-s| \leq h}\left\|Y_{t}-Y_{s}\right\|_{U} \leq \sigma(h) .
$$

If

$$
\int_{0}^{\widehat{\varepsilon}_{0}}\left(\ln \left(1+\frac{3(b-a)}{2 \sigma^{(-1)}(u)}\right)\right)^{1 / 2} d u<\infty
$$


where $\sigma^{(-1)}(u)$ is the inverse function to $\sigma(u)$ and $\widehat{\varepsilon}_{0}=\sigma(b-a)$, then

$$
\sup _{t \in[a, b]}\left|Y_{t}\right| \in L_{U}(\Omega)
$$

and

$$
\left\|\sup _{t \in[a, b]}\left|Y_{t}\right|\right\|_{U} \leq K_{1}:=\inf _{t \in \mathbb{T}}\left\|Y_{t}\right\|_{U}+\frac{e^{2}}{\theta(1-\theta)} \int_{0}^{\theta \widehat{\varepsilon}_{0}}\left(\ln \left(1+\frac{3}{2} \frac{b-a}{\sigma^{(-1)}(u)}\right)\right)^{1 / 2} d u .
$$

Here $\theta$ is an arbitrary number such that

$$
0<\theta<\min \left\{1, \frac{\sigma\left(\frac{2(b-a)}{e^{2}-1}\right)}{\sigma(b-a)}\right\} .
$$

Moreover

$$
\mathrm{P}\left\{\sup _{t \in[a, b]}\left|Y_{t}\right|>\varepsilon\right\} \leq 2 \exp \left\{-\frac{\varepsilon^{2}}{K_{1}^{2}}\right\}
$$

for all $\varepsilon>0$.

Proof. The proof follows from Theorem 5.1 for $\mathbb{T}=[a, b]$.

Indeed, the process $Y_{t}$ is separable in the space $\left([a, b], \rho_{Y}\right)$ by condition (38), where

$$
\rho_{Y}(t, s)=\left\|Y_{t}-Y_{s}\right\|_{U}
$$

It is easy to see that

$$
N(u) \leq \frac{b-a}{2 \sigma^{(-1)}(u)}+1
$$

If $u$ is such that $0<u<\widehat{\varepsilon}_{0}$ or, equivalently, if

$$
\frac{b-a}{\sigma^{(-1)}(u)} \geq 1
$$

then

$$
N(u) \leq \frac{3}{2} \frac{b-a}{\sigma^{(-1)}(u)}
$$

Thus

$$
\int_{0}^{\theta \widehat{\varepsilon}_{0}}(\ln (1+N(u)))^{1 / 2} d u \leq \int_{0}^{\theta \widehat{\varepsilon}_{0}}\left(\ln \left(1+\frac{3}{2} \frac{b-a}{\sigma^{(-1)}(u)}\right)\right)^{1 / 2} d u .
$$

According to Remark [5.1] the condition $N\left(\theta \widehat{\varepsilon}_{0}\right)>e^{2}-1$ can be reduced to the condition

$$
2 \frac{(b-a)}{\sigma^{(-1)}\left(\hat{\varepsilon}_{0} \theta\right)}>e^{2}-1,
$$

that is, to inequalities (41). Now inequality (42) follows from (36).

Theorem 5.3. Let the assumptions of Theorem 2.1 hold. Assume that $X_{t}$ is a solution of equation (11), $t \in[0, T]$, and $0 \leq t_{1}<t_{2} \leq T$. Then $\sup _{t_{1} \leq t \leq t_{2}}\left|X_{t}\right| \in L_{U}(\Omega)$ and

$$
\begin{aligned}
& \left\|\sup _{t_{1} \leq t \leq t_{2}}\left|X_{t}\right|\right\|_{U} \\
& \quad \leq \sqrt{2}(R+1) B+e^{2}\left(\frac{3}{2}\right)^{\gamma / 2} \frac{H F_{7}}{\gamma(H-\gamma / 2)} \theta^{-\gamma /(2 H)}(1-\theta)^{-1}\left(t_{2}-t_{1}\right)^{H}=: L,
\end{aligned}
$$

where the constants $R$ and $B$ are defined in Theorem 4.2, $0<\theta<\left(2 /\left(e^{2}-1\right)\right)^{H}$, and $0<\gamma<2 H$. 
Moreover

$$
\mathrm{P}\left\{\sup _{t_{1} \leq t \leq t_{2}}\left|X_{t}\right| \geq \varepsilon\right\} \leq 2 \exp \left\{-\frac{\varepsilon^{2}}{L^{2}}\right\}
$$

for all $\varepsilon>0$.

Proof. We apply Theorem 5.2, The stochastic process $X_{t}$ is almost surely continuous; thus it is separable. Set $\sigma(h)=F_{7} h^{H}$ in Theorem 5.2. If $\widehat{\varepsilon}_{0}=\sigma\left(t_{2}-t_{1}\right)$, then it is easy to see that

$$
\begin{aligned}
I\left(\theta \widehat{\varepsilon}_{0}\right) & :=\int_{0}^{\theta \widehat{\varepsilon}_{0}}\left(\ln \left(1+\frac{3}{2} \frac{t_{2}-t_{1}}{\sigma^{(-1)}(u)}\right)\right)^{1 / 2} d u \\
& =\int_{0}^{\sigma^{(-1)}\left(\theta \widehat{\varepsilon}_{0}\right)}\left(\ln \left(1+\frac{3}{2} \frac{t_{2}-t_{1}}{v}\right)\right)^{1 / 2} H F_{7} v^{H-1} d v .
\end{aligned}
$$

Since

$$
\ln (1+x)=\frac{1}{\gamma} \ln \left((1+x)^{\gamma}\right) \leq \frac{1}{\gamma} \ln \left(1+x^{\gamma}\right) \leq \frac{x^{\gamma}}{\gamma}
$$

for $0<\gamma \leq 1$ and $x>0$, we obtain from (45) that

$$
\begin{aligned}
I\left(\theta \widehat{\varepsilon}_{0}\right) & \leq\left(\frac{3}{2}\right)^{\gamma / 2} H F_{7} \cdot \frac{1}{\gamma} \int_{0}^{\sigma^{(-1)}\left(\theta \widehat{\varepsilon}_{0}\right)} v^{H-1-\gamma / 2} d v \cdot\left(t_{2}-t_{1}\right)^{\gamma / 2} \\
& =\left(t_{2}-t_{1}\right)^{\gamma / 2}\left(\sigma^{(-1)}\left(\theta \widehat{\varepsilon}_{0}\right)\right)^{H-\gamma / 2} \frac{\left(\frac{3}{2}\right)^{\gamma / 2}}{\gamma} \cdot \frac{H F_{7}}{H-\gamma / 2}
\end{aligned}
$$

for all $0<\gamma<2 H$. It is obvious that

$$
\sigma^{(-1)}\left(\theta \widehat{\varepsilon}_{0}\right)=\theta^{1 / H} \sigma^{(-1)}\left(\widehat{\varepsilon}_{0}\right)=\theta^{1 / H}\left(t_{2}-t_{1}\right) .
$$

Thus

$$
I\left(\theta \widehat{\varepsilon}_{0}\right) \leq\left(t_{2}-t_{1}\right)^{H} \frac{\left(\frac{3}{2}\right)^{\gamma / 2}}{\gamma} \theta^{1-\gamma /(2 H)} \cdot \frac{H F_{7}}{H-\gamma / 2} .
$$

Now the proof of (43) and (44) follows from (45)-(46) and Theorem 5.2.

Remark 5.3. Inequality (43) shows that the estimates for the distribution of the supremum of the process $X_{t}$ have the same order as in the case of Gaussian processes (see, for example, 4]).

Corollary 5.1. Let $X_{t}, t \in[0, T]$, be a solution of equation (11) and let the assumptions of Theorem 2.1 hold for $0 \leq t_{1}<t_{2} \leq T$. Then

$$
\left(\mathrm{E}\left(\sup _{t_{1} \leq t \leq t_{2}}\left|X_{t}\right|\right)^{p}\right)^{1 / p} \leq C_{p} \cdot L
$$

for all $p \geq 1$, where the constant $L$ is defined by (43) and $C_{p}=2^{1 / p} \sqrt{p} / 2$.

Proof. Corollary 5.1 follows from Theorem 5.3. Indeed, it is shown in [6, Lemma 2.3.3] that $\left(\mathrm{E}|\xi|^{p}\right)^{1 / p} \leq C_{p}\|\xi\|_{U}$ for all random variables $\xi \in L_{U}(\Omega)$ where $U(x)=\exp \left\{x^{2}\right\}-1$ and $p \geq 1$. Therefore inequality (47) follows from (43).

Corollary 5.2. Let $X_{t}$ be a solution of equation (11), $t \in[0, T]$, and $0 \leq t_{1}<t_{2} \leq T$. Then

$$
\mathrm{E} \exp \left\{\lambda \sup _{t_{1} \leq t \leq t_{2}}\left|X_{t}\right|\right\} \leq 2 \exp \left\{\frac{\lambda^{2} L^{2}}{4}\right\}
$$

for $\lambda \in \mathbb{R}$, where the constant $L$ is defined by (43).

Corollary 5.2 follows from Theorem 5.2 and Lemma 4.2 . 


\section{The modulus of COntinuity of a SOlution of EQuAtion (1)}

Definition 6.1 ([6]). We say that a $C$-function $U(x)$ (that is, a continuous even convex function such that $U(0)=0$ and $U(x)$ increases in the domain $x>0$ ) satisfies the $\Delta^{2}$ condition if there are constants $x_{0}>0$ and $L_{0}>1$ such that $U^{2}(x) \leq U\left(L_{0} x\right)$ for $x \geq x_{0}$.

Example 6.1 ([6]). The function $\exp \left\{x^{2}\right\}-1$ satisfies the $\Delta^{2}$ condition for $x_{0}=0$ and $L_{0}=\sqrt{2}$.

Theorem 6.1. Let $\left\{X_{t}, t \in \mathbb{T}\right\}$ be a stochastic process belonging to the Orlicz space $L_{U}(\Omega)$. Assume that the function $U(x)$ satisfies $\Delta^{2}$ conditions with constants $x_{0}$ and $L_{0}$. Let $Z_{0}=\max \left(x_{0}, L_{0}\right)$ and let $\rho_{X}(t, s)=\left\|X_{t}-X_{s}\right\|_{U}, t, s \in T$, be the pseudometric generated by the process $X_{t}$. Assume that the space $\left(\mathbb{T}, \rho_{X}\right)$ is separable and that the process $X_{t}$ is separable in the space $\left(\mathbb{T}, \rho_{X}\right)$. Let $\varepsilon_{0}=\sup _{t, s \in T} \rho_{X}(t, s), N(u)$ denote the minimal number of closed balls of radius $u$ that cover $\left(\mathbb{T}, \rho_{X}\right)$, and let $N_{1}(u), u>0$, be a decreasing function such that $N_{1}(u) \geq N(u), u>0$. If

$$
q(\varepsilon):=\int_{0}^{\varepsilon} U^{(-1)}\left(N_{1}(u)\right) d u<\infty
$$

for all $\varepsilon>0$, then

$$
\mathrm{P}\left\{\sup _{0<\rho_{X}(t, s)<\varepsilon} \frac{\left|X_{t}-X_{s}\right|}{C q\left(\rho_{X}(t, s)\right)} \geq x\right\} \leq \frac{3+\sqrt{2}}{U(x)}
$$

for all $\varepsilon \in\left(0, \varepsilon_{0}\right)$ such that $N_{1}(\varepsilon)>U\left(Z_{0}\right)$ and for all $x \geq Z_{0}$. Moreover,

$$
\lim _{\varepsilon \downarrow 0} \sup \frac{\Delta X_{\varepsilon}}{C Z_{0} q(\varepsilon)} \leq 1
$$

with probability one, where

$$
\Delta X_{\varepsilon}=\sup _{\substack{t, s \in T, 0<\rho_{X}(t, s)<\varepsilon}}\left|X_{t}-X_{s}\right|
$$

and $C=3 L_{0}\left(5+4 L_{0}\right)$.

Theorem [6.1 is proved in [6] for $N_{1}(u)=N(u)$. The substitution $N_{1}(u)$ for $N(u)$ does not change the proof essentially.

Corollary 6.1. Let $\left\{X_{t}, t_{1} \leq t \leq t_{2}\right\}$ be a separable stochastic process belonging to the space $L_{U}(\Omega)$ where $U(x)=\exp \left\{x^{2}\right\}-1$. Let

$$
\sup _{\substack{|t-u| \leq h, t, u \in\left[t_{1}, t_{2}\right]}}\left\|X_{t}-X_{u}\right\|_{U} \leq D h^{\alpha}, \quad D>0,0<\alpha \leq 1 .
$$

Then

$$
\mathrm{P}\left\{\sup _{\substack{0<|t-u|<\delta, t, u \in\left[t_{1}, t_{2}\right]}} \frac{\left|X_{t}-X_{u}\right|}{d g\left(D|t-u|^{\alpha}\right)}>x\right\} \leq \frac{3+\sqrt{2}}{U(x)}
$$

for all $x>\sqrt{2}$ and $0<\delta \leq\left(t_{2}-t_{1}\right)^{\alpha} D /\left(2^{\alpha}\left(e^{2}-2\right)^{\alpha}\right)$, where

$$
g(\varepsilon):=\int_{0}^{\varepsilon}\left(\ln \left(\frac{\left(t_{2}-t_{1}\right) D^{1 / \alpha}}{2 u^{1 / \alpha}}+2\right)\right)^{1 / 2} d u
$$


and $d=3 \sqrt{2}(5+4 \sqrt{2})$. Moreover,

$$
\lim _{\delta \downarrow 0} \sup \frac{\Delta X_{\delta}}{d \sqrt{2} g\left(D \cdot \delta^{\alpha}\right)} \leq 1
$$

with probability one, where $\Delta X_{\delta}=\sup _{|t-u|<\delta, t, u \in\left[t_{1}, t_{2}\right]}\left|X_{t}-X_{u}\right|$.

Proof. It follows from Example 6.1 that the $C$-function $U(x)=\exp \left\{x^{2}\right\}-1$ satisfies the $\Delta^{2}$ condition for $x_{0}=0, L_{0}=\sqrt{2}$, and $Z_{0}=\sqrt{2}$. It is also clear that

$$
U^{(-1)}(x)=(\ln (x+1))^{1 / 2}, \quad x>0,
$$

and $q(\varepsilon)=\int_{0}^{\varepsilon}\left(\ln \left(N_{1}(u)+1\right)\right)^{1 / 2} d u$. Since

$$
N(u) \leq \frac{\left(t_{2}-t_{1}\right) D^{1 / \alpha}}{2 u^{1 / \alpha}}+1
$$

one can choose

$$
N_{1}(u)=\frac{\left(t_{2}-t_{1}\right) D^{1 / \alpha}}{2 u^{1 / \alpha}}+1
$$

Thus

Therefore

$$
q(\varepsilon)=\int_{0}^{\varepsilon}\left(\ln \left(\frac{\left(t_{2}-t_{1}\right) D^{1 / \alpha}}{2 u^{1 / \alpha}}+2\right)\right)^{1 / 2} d u=g(\varepsilon) .
$$

$$
\sup _{0<|t-u|<\delta} \frac{\left|X_{t}-X_{u}\right|}{g\left(D|t-u|^{\alpha}\right)} \leq \sup _{0<|t-u|<\delta} \frac{\left|X_{t}-X_{u}\right|}{g\left(\rho_{X}(t, u)\right)} \leq \sup _{0<\rho_{X}(t, u)<D \delta^{\alpha}} \frac{\left|X_{t}-X_{u}\right|}{g\left(\rho_{X}(t, u)\right)} .
$$

Now inequality (51) follows from (50), since the process $X_{t}$ is separable and (50) implies that $X_{t}$ is separable in the space $\left(\mathbb{T}, \rho_{X}\right)$ for $\mathbb{T}=\left[t_{1}, t_{2}\right]$. Inequality (52) can be proved analogously. Necessary restrictions for $\delta$ follow from

$$
N_{1}(\delta)>U\left(Z_{0}\right)=e^{2}-1 .
$$

The latter corollary implies the following result.

Theorem 6.2. Let $\left\{X_{t}, t \in[0, T]\right\}$ be a solution of equation (1) and let the assumptions of Theorem 4.4 hold. If $h(y):=\int_{0}^{y}\left(\ln \left(v^{-1 / H}+2\right)\right)^{1 / 2} d v, y>0$, then

$$
\mathrm{P}\left\{\sup _{\substack{0<|t-u|<\delta, t, u \in\left[t_{1}, t_{2}\right]}} \frac{\left|X_{t}-X_{u}\right|}{d D 2^{-H}\left(t_{2}-t_{1}\right)^{H} h\left(\frac{|t-u|^{H} 2^{H}}{\left(t_{2}-t_{1}\right)^{H}}\right)}>x\right\} \leq \frac{3+\sqrt{2}}{U(x)}
$$

for all $x \geq \sqrt{2}, 0 \leq t_{1}<t_{2} \leq T$, and $\delta \leq\left(t_{2}-t_{1}\right)^{H} D /\left(2^{H}\left(e^{2}-2\right)^{H}\right)$, where $D=\sqrt{2} F_{7}$. Moreover,

$$
\lim _{\delta \downarrow 0} \sup \frac{\sup _{\substack{|t-u|<\delta, t, u \in\left[t_{1}, t_{2}\right]}}\left|X_{t}-X_{u}\right|}{\sqrt{2} d D\left(t_{2}-t_{1}\right)^{H} h\left(\frac{\delta^{H} 2^{H}}{\left(t_{2}-t_{1}\right)^{H}}\right) 2^{-H}} \leq 1
$$

with probability one.

Theorem 6.2 follows from Theorems 4.3 and 4.4 and Corollary 6.1 (inequality (52)). Indeed, $\alpha=H, D=\sqrt{2} F_{7}$, and

$$
g\left(F_{7} \sqrt{5}|t-u|^{H}\right)=h\left(|t-u|^{H} \frac{2^{H}}{\left(t_{2}-t_{1}\right)^{H}}\right) D \frac{\left(t_{2}-t_{1}\right)^{H}}{2^{H}}
$$

in the case of Theorem 6.2. 
Definition 6.2. Let $(\mathbb{T}, \rho)$ be a metric space and let the $q=\{q(t), t>0\}$ be a modulus of continuity (that is, a positive continuous function such that $q(0)=0$ and

$$
q(t+s) \leq q(t)+q(s)) .
$$

The family of functions $y_{t}, t \in \mathbb{T}$, such that

$$
\sup _{\substack{t, s \in \mathbb{T} \\ t \neq s}} \frac{\left|y_{t}-y_{s}\right|}{q(\rho(t, s))}<\infty
$$

is called the Lipschitz space $\Lambda_{q}(\mathbb{T}, \rho)$.

Remark 6.1. Theorem 6.2 claims that a solution of equation (1) belongs to the space $\Lambda_{q}(\mathbb{T}, \rho)$ with probability one, where $\mathbb{T}=\left[t_{1}, t_{2}\right], \rho(t, s)=|t-s|$, and

$$
q(y)=h\left(\frac{y^{H} 2^{H}}{\left(t_{2}-t_{1}\right)^{H}}\right) \text {. }
$$

Inequality (53) provides a bound for the distribution of the norm of $X_{t}$ in this space.

Corollary 6.2. Let $X_{t}$ be a solution of equation (11) and let the assumptions of Theorem 6.2 hold. Then for all $0<\gamma<2 H$ the process $X_{t}$ belongs to the space $\Lambda_{q}(\mathbb{T}, \rho)$ with probability one, where $\mathbb{T}=\left[t_{1}, t_{2}\right], 0 \leq t_{1}<t_{2} \leq T, \rho(s, u)=|s-u|$, and

$$
q(|s-u|)=C_{\gamma}|s-u|^{H-\gamma / 2}, \quad C_{\gamma}=d D 2^{-\gamma / 2} \gamma^{-1 / 2} \frac{4 H-\gamma}{2 H-\gamma}\left(t_{2}-t_{1}\right)^{\gamma / 2} .
$$

Moreover

$$
\mathrm{P}\left\{\sup _{\substack{0<|t-u|<\delta, t, u \in\left[t_{1}, t_{2}\right]}} \frac{\left|X_{t}-X_{u}\right|}{C_{\gamma}|t-u|^{H-\gamma / 2}}>x\right\} \leq \frac{3+\sqrt{2}}{U(x)}
$$

if $x>\sqrt{2}$ and $\delta$ are as in Theorem 6.2.

Proof. The inequality $\ln (1+x) \leq x^{\gamma} / \gamma$ for $x>0$ and $0<\gamma \leq 1$ easily implies for $2 \delta<t_{2}-t_{1}$ that

$$
\begin{aligned}
h\left(\delta^{H} \frac{2^{H}}{\left(t_{2}-t_{1}\right)^{H}}\right) & \leq \int_{0}^{\left(\frac{2 \delta}{t_{2}-t_{1}}\right)^{H}}\left(\ln \left(v^{-1 / H}+2\right)\right)^{1 / 2} d v \leq \int_{0}^{\left(\frac{2 \delta}{t_{2}-t_{1}}\right)^{H}} \frac{1+v^{-\gamma /(2 H)}}{\gamma^{1 / 2}} d v \\
& \leq \gamma^{-1 / 2}\left(\frac{2 \delta}{t_{2}-t_{1}}\right)^{H}+\frac{\gamma^{-1 / 2}}{1-\frac{\gamma}{2 H}}\left(\frac{2 \delta}{t_{2}-t_{1}}\right)^{H-\gamma / 2} \\
& \leq \gamma^{-1 / 2} \frac{4 H-\gamma}{2 H-\gamma}\left(\frac{2 \delta}{t_{2}-t_{1}}\right)^{H-\gamma / 2} .
\end{aligned}
$$

The remaining part of the proof follows from (53).

\section{BIBLIOGRAPHY}

1. D. Nualart and Y. Ouknine, Regularization of differential equations by fractional noise, Stoch. Process. Appl. 102 (2002), 103-116. MR1934157 (2004b:60151)

2. Yu. V. Kozachenko and Yu. S. Mishura, Maximal upper bounds for moments of stochastic integrals and solutions of stochastic differential equations with respect to the fractional Brownian motion with Hurst index $H<1 / 2$. I, Teor. Imovirnost. ta Matem. Statyst. 75 (2006), 45-56; English transl. in Theory Probab. Math. Statist. 75 (2007). MR2321180 (2008g:60167)

3. S. G. Samko, A. A. Kilbas, and O. I. Marichev, Fractional Integrals and Derivatives. Theory and Applications, "Nauka i tekhnika", Minsk, 1987; English transl., Gordon and Breach Science Publishers, New York, 1993. MR1347689(96d:26012) 
YU. V. KOZACHENKO AND YU. S. MISHURA

4. X. Fernique, Regularité des trajectoires des fonctions aléatoires gaussiennes. École d'Été de Probabilités de Saint-Flour IV, Lecture Notes in Mathematics 480, Springer, Berlin, 1975, 2-95. MR0413238 (54:1355)

5. J. Mémin, Yu. Mishura, and E. Valkeila, Inequalities for the moments of Wiener integrals with respect to fractional Brownian motion, Stat. Prob. Letters 51 (2001), 197-206. MR1822771 (2002b:60096)

6. V. V. Buldygin and Yu. V. Kozachenko, Metric Characterization of Random Variables and Random Processes, American Mathematical Society, Providence, Rhode Island, 2000. MR1743716 (2001g:60089)

Department of Probability Theory and Mathematical Statistics, Faculty for Mechanics and Mathematics, National Taras Shevchenko University, Academician Glushkov Avenue 6 , KYIV 03127, UKRAINE

E-mail address: yvk@univ.kiev.ua

Department of Probability Theory and Mathematical Statistics, Faculty for Mechanics and Mathematics, National Taras Shevchenko University, Academician Glushkov Avenue 6 , KYIV 03127, UKRAINE

E-mail address: myus@univ.kiev.ua

Received 2/OCT/2006

Translated by O. I. KLESOV 